TRANSACTIONS OF THE

AMERICAN MATHEMATICAL SOCIETY

Volume 355, Number 9, Pages 3651-3667

S 0002-9947(03)03280-X

Article electronically published on March 17, 2003

\title{
COMPLETE HOMOGENEOUS VARIETIES: STRUCTURE AND CLASSIFICATION
}

\author{
CARLOS SANCHO DE SALAS
}

\begin{abstract}
Homogeneous varieties are those whose group of automorphisms acts transitively on them. In this paper we prove that any complete homogeneous variety splits in a unique way as a product of an abelian variety and a parabolic variety. This is obtained by proving a rigidity theorem for the parabolic subgroups of a linear group. Finally, using the results of Wenzel on the classification of parabolic subgroups of a linear group and the results of Demazure on the automorphisms of a flag variety, we obtain the classification of the parabolic varieties (in characteristic different from 2,3). This, together with the moduli of abelian varieties, concludes the classification of the complete homogeneous varieties.
\end{abstract}

\section{INTRODUCTION}

Let $X$ be a variety over an algebraically closed field $k$. Let $G$ be the functor of automorphisms of $X$ that acts naturally on $X$. We say that $X$ is homogeneous if $G$ acts transitively on $X$, that is, if for each pair of points $x, x^{\prime} \in \operatorname{Hom}(S, X)$, there exists an automorphism $\tau \in \operatorname{Aut}_{S}(X \times S)$ (after a faithfully flat base change on $S$ ) transforming one into the other: $\tau(x)=x^{\prime}$.

It is known that the group of automorphisms of a complete variety exists, i.e., the functor $G$ is representable, and it is locally of finite type (see 7]). Hence, if $X$ is smooth, connected and homogeneous, then the reduced connected component through the origin of $G$ acts transitively on $X$. Therefore, every smooth and connected homogeneous variety is isomorphic to $G / P$, with $G$ a smooth and connected algebraic group and $P \subset G$ a subgroup.

Let $\operatorname{Aut}^{0}(X)$ denote the reduced connected component through the origin of the automorphism scheme of $X$.

The main results that we obtain here are:

(1) A complete homogeneous variety splits canonically and uniquely as a direct product of an abelian variety and a parabolic variety.

A parabolic variety means a variety of the form $G / P$ with $G$ an affine, smooth and connected algebraic group and $P$ a parabolic subgroup (eventually not reduced), i.e., a subgroup containing a Borel subgroup of $G$. This is Theorem 5.2 The analogue of this result for compact Kähler manifolds is due to A. Borel and R. Remmer (see [2]).

Received by the editors February 15, 2002 and, in revised form, October 11, 2002.

2000 Mathematics Subject Classification. Primary 14M17, 14M15, 14L30, 32M10.

This research was partially supported by the Spanish DGI through research project BFM20001315 and by the "Junta de Castilla y León" through research project SA009/01. 
For the proof, it is necessary to prove that any action of an affine group on a variety with projective orbits is trivial. More precisely, it is proved that if an affine algebraic group acts on a variety and the orbits are projective and equidimensional, then the variety splits as the direct product of an orbit and the quotient by the action. This is Theorem 3.3 Borel proved an analogue of this result for a complex variety (see [1]).

(2) From this result the next one, of great interest for the classification, follows easily: The automorphism group of a homogeneous complete variety classifies (modulo isomorphisms) the variety, up to the choice, in such a group, of a class (modulo automorphisms of the group) of a parabolic subgroup $[P]$. Moreover, this group of automorphisms splits, in a unique way, as the direct product of a semisimple group of adjoint type (that is, with trivial center) and an abelian variety, and the subgroup $P$ is a parabolic subgroup of the semisimple part. That is, to each homogeneous variety $X$ is assigned its automorphism group $\operatorname{Aut}^{0}(X)=G \times A$ and the class $[P]$, modulo automorphisms, of a parabolic subgroup $P \subset G$; thus the maps $X \mapsto(G, A,[P])$ and $(G, A,[P]) \mapsto X=G / P \times A$ establish an equivalence of objects modulo isomorphisms. This is Theorem 5.7 .

It should be noted that the triplets $(G, A,[P])$ are chosen with the single condition that $\operatorname{Aut}^{0}(G / P)=G$. Therefore, a first question is to know whether $G$ may be any semisimple group of adjoint type; in other words, if $G$ is given, does there exist a parabolic subgroup $P \subset G$ such that $\operatorname{Aut}^{0}(G / P)=G$ ? The answer is affirmative, and is due to Demazure $([4])$. From his results one obtains in particular:

If $G$ is a semisimple group of adjoint type and $\mathcal{B}$ is the variety of its Borel subgroups, then $\operatorname{Aut}^{0}(\mathcal{B})=G$.

In conclusion, the classification of homogeneous complete varieties is equivalent to the classification of abelian varieties, semisimple groups of adjoint type (both of them well studied, see [8], [9], 6] ) and parabolic subgroups $P$ of a given semisimple group $G$ (modulo automorphisms) such that $\operatorname{Aut}^{0}(G / P)=G$. Therefore, the classification is essentially reduced to the classification of (non-exceptional, in the sense of Demazure 4]) parabolic subgroups of a simple group of adjoint type.

Finally, from the classification of the parabolic subgroups (in characteristic $p \neq$ 2,3 ) due to Wenzel (12]) and the determination of the non-exceptional and reduced parabolic subgroups due to Demazure (4]), we shall classify the parabolic varieties in characteristic $p \neq 2,3$.

\section{KNOWN RESULTS}

The following results are well known and may be found in [5], 6], 8], 9], [11].

Theorem 1.1 (Barsotti-Chevalley). Let $\bar{G}$ be a smooth and connected algebraic group. There exists a unique normal subgroup $G \subset \bar{G}$ that is affine, smooth and connected, such that the quotient $\bar{G} / G$ is an abelian variety.

Definition 1.2. An affine algebraic group is said to be linearly reductive if any linear representation splits as a direct sum of simple representations.

An example of a linearly reductive group is the torus, and this is the only smooth and connected one in positive characteristic.

Theorem 1.3. If a linearly reductive group acts on a smooth variety $X$, then the closed subscheme $X^{G}$ of the fixed points is a smooth subscheme whose tangent space at each point $x$ is the subspace of the $G$-invariant tangent vectors of $X$ at $x$. 
Definition 1.4. A parabolic subgroup of an affine, smooth and connected group $G$ is a subgroup $P \subset G$ such that the quotient variety $G / P$ is complete.

Definition 1.5. A parabolic variety is a complete variety of the form $G / P$, where $G$ is an affine, smooth and connected group and $P \subset G$ is a subgroup. A parabolic variety is called a flag variety if $P$ is a reduced scheme.

Theorem 1.6 (Chevalley). If $G$ is an affine group and $H \subset G$ a subgroup, then $G / H$ is a quasiprojective scheme and the action of $G$ on $G / H$ is projective, i.e., there exists a linear representation $E$ of $G$ such that $G / H$ is a sub-G-scheme of $\mathbb{P}(E)$.

Remark 1.7. It follows from this theorem that any parabolic variety is projective.

Theorem 1.8. Any flag variety is a Fano variety; that is, the dual of the dualizing sheaf is ample.

Theorem 1.9. Let $G$ be a semisimple group, Aut $G$ the automorphism group of $G$, and $I \subset$ Aut $G$ the subgroup of the inner automorphisms of $G$. Then (Aut $G$ ) $/ I$ is finite. In particular, if $G$ is of adjoint type, then the connected component through the origin of Aut $G$ is isomorphic to $G$.

Theorem 1.10. Let $X$ be an abelian variety, and $\operatorname{Aut}^{0} X \subset$ Aut $X$ the connected component through the origin of the automorphism group of $X$ (as a variety). The inclusion $X \hookrightarrow \mathrm{Aut}^{0} X, x \mapsto t_{x}=$ translation by $x$, is an isomorphism.

Theorem 1.11 (Borel's fixed point theorem). If a smooth, solvable and connected affine group acts on a complete variety, then the subscheme of the fixed points is not empty.

Definition 1.12. We shall denote by $\operatorname{Pic}(X)$ the scheme in groups (if it exists) that parametrizes the invertible sheaves on $X$ and by $P i c^{0}(X)$ the connected component of $\operatorname{Pic}(X)$ through the origin.

Theorem 1.13. If $X$ is a complete variety, then Pic $(X)$ exists; it is a proper scheme and the tangent space at the origin is isomorphic to $H^{1}\left(X, \mathcal{O}_{X}\right)$. Moreover,

(1) If $X$ is an abelian variety, then Pic $(X)$ is smooth; hence, $P_{i c}^{0}(X)$ is also an abelian variety.

(2) If $X$ is a parabolic variety, then $\operatorname{Pic}^{0}(X)=0$; in particular, Pic $(X)$ is a discrete $k$-scheme (i.e., ЏSpec $k$ ). Furthermore, if $X=G / P$ where $G$ is semisimple and simply connected, then Pic $(X)$ is isomorphic to the character group of $P$.

Let $X$ be a variety such that $\operatorname{Pic}(X)$ exists. Let $\mathcal{P}$ be a universal invertible sheaf on $X \times P i c^{0}(X)$, i.e., an invertible sheaf such that the pair $\left(\operatorname{Pic}^{0}(X), \mathcal{P}\right)$ represents the functor of invertible sheaves of degree zero on $X$. By the universal property, $\mathcal{P}$ is univocally determined up to inverse images of invertible sheaves (of degree $0)$ on $P i c^{0}(X)$ by the projection $X \times \operatorname{Pic}^{0}(X) \rightarrow P i c^{0}(X)$. Hence, if one fixes a point $x_{0} \in X$ and requires that $\mathcal{P}$ be trivial on $x_{0} \times P i c^{0}(X)$, then $\mathcal{P}$ is completely determined.

Given $\mathcal{P}$, one defines $\varphi: X \rightarrow \operatorname{Pic}^{0}\left(\operatorname{Pic}^{0}(X)\right)$ by $\varphi(x)=\mathcal{P}_{\left.\right|_{x \times P i c}(X)}$. If, in order to determine $\mathcal{P}$, one replaces the chosen point $x_{0}$ by another one, $x_{0}^{\prime}$, then one obtains the same morphism composed with the translation on $\operatorname{Pic}^{0}\left(\operatorname{Pic}^{0}(X)\right)$ by 
the invertible sheaf $\varphi\left(x_{0}^{\prime}\right)^{-1}$. That is, $\varphi$ is univocally determined, up to translations on $\operatorname{Pic}^{0}\left(P i c^{0}(X)\right)$, by the choice of a point of $X$. If $X$ is a group, one chooses as $x_{0}$ the origin of the group; this is equivalent to saying that $\varphi$ is a morphism of groups $(\varphi(0)=0)$.

Theorem 1.14 (Duality of abelian varieties). If $A$ is an abelian variety, then the natural morphism $A \rightarrow \operatorname{Pic}^{0}\left(\operatorname{Pic}^{0}(A)\right)$ is an isomorphism.

Theorem 1.15. If $X, Y$ are complete varieties, then

$$
\operatorname{Pic}^{0}(X) \times \operatorname{Pic}^{0}(Y)=\operatorname{Pic}^{0}(X \times Y),
$$

and the correspondence is $\left(\mathcal{L}, \mathcal{L}^{\prime}\right) \mapsto \mathcal{L} \underset{k}{\otimes} \mathcal{L}^{\prime}$.

\section{LOCAL TRIVIALITY OF DEFORMATIONS OF PARABOLIC SUBGROUPS}

Let us assume that $k$ is an algebraically closed field of arbitrary characteristic $p$, and let $G$ be an affine, smooth and connected algebraic group over $k$.

Definition 2.1. The nilpotence degree of an algebraic group $G$ (over an algebraically closed field) is the order of the quotient by its reduced subgroup: $\left|G / G_{\text {red }}\right|$. That is, it is the dimension of the finite vector space of the functions of $G / G_{r e d}$.

It is known that a subgroup $P \subset G$ is parabolic if and only if it contains a Borel subgroup $B$ of $G$. Moreover, given a Borel subgroup $B$, each parabolic subgroup has a conjugated one containing $B$, because all Borel subgroups are conjugate. Therefore, the problem is to determine the structure of the parabolic subgroups containing a given Borel subgroup.

The aim of this section is to prove the local triviality of any deformation of parabolic subgroups (containing a Borel subgroup $B$ ). More precisely, let us denote $X_{S}=X \times S$ for each $k$-scheme $S$. Then,

Theorem 2.2. Let $S$ be a connected scheme and $\widetilde{P} \subset G_{S}$ a subscheme in groups over $S$ containing $B_{S}$. Then:

(1) If $\widetilde{P}$ is flat over $S$, then it is constant; that is, $\widetilde{P}=P_{S}$ for some subgroup $P \subset G$ containing $B$.

(2) If $S$ is integral and the fibres of $\widetilde{P} \rightarrow S$ have constant dimension and nilpotence degree, then $\widetilde{P}$ is constant: $\widetilde{P}=P_{S}$.

One first observes that any parabolic subgroup contains the radical $R(G)$ of the group (the maximal solvable, normal, smooth and connected subgroup of $G$ ). Hence, the parabolic subgroups are in biunivocal correspondence with the parabolic subgroups of the quotient $G / R(G)$, which is a semisimple group. Therefore, we shall assume in the following that $G$ is semisimple.

Let $T \subset B$ be a maximal torus of $G, \mathcal{R}$ the roots system of $G$ associated with $T, \mathcal{R}^{+}$the positive roots (i.e., the roots of $(B, T)$ ) and $\mathcal{S} \subset \mathcal{R}$ the basis of simple roots contained in $\mathcal{R}^{+}$.

Remark 2.3. The reduced parabolic subgroups (containing $B$ ) are in biunivocal correspondence with the subsets $I$ of the basis $\mathcal{S}$. In particular, the number of them is finite (exactly $2^{|\mathcal{S}|}$ ). Therefore, the theorem is almost immediate in characteristic 0 , since any group is reduced, and hence the scheme of parabolic subgroups containing $B$ is finite and discrete. Thus, for the proof of the theorem, we shall 
assume that the characteristic is positive, $p>0$, although the same proof is valid in characteristic 0 with the corresponding simplifications.

Let $\mathcal{B}$ be the variety of Borel subgroups of $G$. It is known that $\mathcal{B}=G / B$, as $G$ varieties. Let $U \subset B$ be the unipotent part of $B$ and $U^{-}$the unipotent part of the Borel subgroup $B^{-}$opposite to $B$ (that is, the unique Borel subgroup containing $T$ and such that $\left.B \cap B^{-}=T\right)$. One has that $U^{-}$is identified, as a $U^{-}$-scheme, with an open subset $\mathcal{U}^{-}$of $\mathcal{B}$ (precisely, with the open set of the Borel subgroups $B^{\prime}$ such that $B^{\prime} \cap U^{-}=\{e\}$ ), which coincides with the unique open orbit under the action of $B^{-}$.

Lemma 2.4. Let $S \rightarrow$ Spec $k$ be a base change. The map $\widetilde{P} \mapsto \widetilde{P} \cap U_{S}^{-}$, between the subschemes in groups of $G_{S}$ over $S$ containing $B_{S}$ and the subschemes in groups of $U_{S}^{-}$over $S$ stable by $T$, is injective. Moreover, flat subschemes correspond to flat subschemes.

Proof. Each subscheme in groups of $G_{S}, \widetilde{P} \supset B_{S}$, defines a closed subscheme $\widetilde{P} / B_{S} \subset(G / B)_{S}=\mathcal{B}_{S}$, which determines $\widetilde{P}$, since $\widetilde{P}$ is the preimage of that closed subscheme by the morphism to the quotient $\pi: G_{S} \rightarrow G_{S} / B_{S}=\mathcal{B}_{S}$. Moreover, $\mathcal{U}_{S}^{-} \cap\left(\tilde{P} / B_{S}\right)$ is dense in $\tilde{P} / B_{S}$ (because it is dense in the fibres over $S$, since any parabolic subgroup is irreducible). Hence, $\widetilde{P} / B_{S}$ is the closure of $\mathcal{U}_{S}^{-} \cap\left(\widetilde{P} / B_{S}\right)$ in $\mathcal{B}_{S}$. It follows that the closed subscheme $\mathcal{U}_{S}^{-} \cap\left(\tilde{P} / B_{S}\right) \subset \mathcal{U}_{S}^{-}$determines $\widetilde{P}$. However, the identification of $U^{-}$with $\mathcal{U}^{-}$induces another one of $U_{S}^{-}$with $\mathcal{U}_{S}^{-}$, and it is clear that $\mathcal{U}_{S}^{-} \cap\left(\tilde{P} / B_{S}\right)$ is identified with $U_{S}^{-} \cap \tilde{P}$, which is a subscheme in groups of $U_{S}^{-}$and is stable under the action of $T$ by conjugation (since both subschemes are stable). In conclusion, $U_{S}^{-} \cap \tilde{P}$ determines $\widetilde{P}$. The flatness of $\widetilde{P} \cap U_{S}^{-}$when $\widetilde{P}$ is flat follows from the flatness of $\widetilde{P} / B_{S} \subset \mathcal{B}_{S}$ (this one is flat by descent, since $\widetilde{P} \rightarrow \tilde{P} / B_{S}$ is faithfully flat), since $\widetilde{P} \cap U_{S}^{-}$is identified with the open subscheme $\left(\tilde{P} / B_{S}\right) \cap \mathcal{U}_{S}^{-}$.

In the case that $S=\operatorname{Spec} k$, this lemma may be obtained from Proposition 4 of [12] as a particular case. Therefore, this lemma generalizes that proposition for the relative case.

Let $\left\{\alpha_{1}, \ldots, \alpha_{s}\right\}=R^{-}$be the roots corresponding to $B^{-}$. By definition, these are the characters of $T$ appearing in the action of $T$ on the tangent space of $U^{-}$ (so $s=\operatorname{dim} U^{-}$). They are nontrivial characters, and none of the $\alpha_{i}$ is a power of $\alpha_{j}$ for $i \neq j$ (in particular, they are different). Moreover, for each $\alpha_{i}$ there exists a unique additive subgroup $G_{a}^{\alpha_{i}} \subset U^{-}$stable by $T$ and such that $T$ acts by multiplication by the character $\alpha_{i}$, in such a way that the multiplication morphism $G_{a}^{\alpha_{1}} \times \cdots \times G_{a}^{\alpha_{s}} \rightarrow U^{-}$is an isomorphism of $T$-varieties.

If $\widetilde{P} \subset G_{S}$ is a parabolic subgroup containing $B$, then, in particular, $\widetilde{P} \cap U_{S}^{-}=H$ is a subgroup of $U_{S}^{-}$stable under the action of $T$ by conjugation. Therefore, it suffices to study the structure of these subgroups.

Definition 2.5. A cone is the spectrum of an $\mathbb{N}$-graded algebra.

One observes that a $\mathbb{Z}$-graduation on an algebra $A$ is equivalent to an action of algebras of the multiplicative group $G_{m}$ on $A$. 
Definition 2.6. We say that an action of $G_{m}$ on $\operatorname{Spec} A$ is conic if the corresponding graduation on $A$ is an $\mathbb{N}$-graduation.

Proposition 2.7. If $G_{m}$ acts on an affine scheme $X$ in a conic way and $Y \subset X$ is a stable closed subscheme, then the action of $G_{m}$ on $Y$ is conic.

Proof. Immediate.

Remark 2.8. The action of the maximal torus $T$ on $U^{-}$(with the above notation) is conic for certain one-parameter subgroups $G_{m} \hookrightarrow T$.

Lemma 2.9. The system of negative roots can be ordered in such a way that for each $i \leq s$ there exists a multiplicative subgroup $G_{m}^{(i)} \subset T$ such that the restriction of the root $\alpha_{j}$ to $G_{m}^{(i)}$ is a nontrivial negative character for $j>i$ and is the trivial character for $j=i$.

Proof. Using the additive notation for the characters of $T$, one has that $R^{-} \subset$ $X(T)=\mathbb{Z} \oplus \stackrel{d}{ } \oplus \oplus \mathbb{Z}$; thus, $R^{-}$are vectors with negative coordinates. One then has to find linear forms $\omega_{i}: X(T) \rightarrow \mathbb{Z}$ (which correspond to multiplicative subgroups of $T$ ) such that $\omega_{i}\left(\alpha_{j}\right)$ are negative for $j>i$ and $\omega_{i}\left(\alpha_{i}\right)=0$, for a certain order of the indices. By recurrence, it clearly suffices to prove that for any subset $J=\left\{\beta_{1}, \ldots, \beta_{r}\right\} \subset R^{-}$there exists a reordering of the indices and a linear form $\omega: X(T) \rightarrow \mathbb{Z}$ such that $\omega$ is negative on $\beta_{j}$ for $j>1$ and $\omega\left(\beta_{1}\right)=0$. Since $R^{-}$is contained in an open half-plane of the corresponding real vector space, any $J \subset R^{-}$ generates a convex cone that contains no full line. Let $\beta_{1} \in J$ generate an extremal ray of that cone. Then there exist a linear form $\omega$ vanishing at $\beta_{1}$, and strictly negative on all other $\beta \in J$ (since no negative roots are proportional); we may further assume that $\omega$ is rational, and even integral.

Corollary 2.10. Let us fix an ordering of the roots given by the preceding proposition. If we denote $U_{i}^{-}=G_{a}^{\alpha_{i}} \times \cdots \times G_{a}^{\alpha_{s}}$, then

- $U_{i}^{-}$is closed in $U^{-}$and stable under the action of $T$;

- $G_{m}^{(i)}$ acts in a conic way on $U_{i}^{-}$, and the subscheme of the fixed points is $\left(U_{i}^{-}\right)^{G_{m}^{(i)}}=G_{a}^{\alpha_{i}}$.

\section{Proposition 2.11.}

(1) Let $H$ be an affine $S$-scheme in groups on which $G_{m}$ acts in a conic way through automorphisms of $S$-schemes in groups. Then, there exists a unique retract $\rho: H \rightarrow H^{G_{m}}$ of $G_{m}$-schemes, where $H^{G_{m}}$ is the closed subscheme in groups of the fixed points of $H$ by $G_{m}$. Moreover, $\rho$ is a morphism of $S$-schemes in groups, and hence $\operatorname{ker} \rho=N$ is the unique subscheme in groups of $H$ stable by $G_{m}$ such that

$$
H=N \underset{S}{\rtimes} H^{G_{m}} .
$$

(2) If $H$ is an affine $S$-scheme in groups on which $G_{m}$ acts in a conic way through automorphisms of $S$-schemes in groups and $\bar{H} \subset H$ is a subscheme in groups stable by $G_{m}$, then $G_{m}$ acts in a conic way on $\bar{H}$ and the restriction of the retract $\rho: H \rightarrow H^{G_{m}}$ to $\bar{H}$ is the corresponding retract $\rho_{\bar{H}}: \bar{H} \rightarrow \bar{H}^{G_{m}} \subset H^{G_{m}}$.

Proof. (1) By the uniqueness of $\rho$ it suffices to prove the statement locally on $S$. That is, we may assume $S=\operatorname{Spec} k$ with $k$ a ring, and $H=\operatorname{Spec} A$, with $A$ a Hopf $k$-algebra. 
By hypothesis, $A$ is a Hopf $T$-algebra, i.e., an $\mathbb{N}$-graded Hopf $k$-algebra. Let us consider the Hopf subalgebra $A_{0} \subset A$ of the elements of degree zero. Let $I \subset A$ be the irrelevant ideal. One has that $I$ is the ideal of a subgroup of $H$ over $k$ and $A_{0} \stackrel{\pi}{\simeq} A / I$ as Hopf $k$-algebras. This means that the inclusion $\operatorname{Spec} A / I \subset \operatorname{Spec} A$ has the retract $\pi_{0}: \operatorname{Spec} A \rightarrow \operatorname{Spec} A_{0}$. Now, $\operatorname{Spec} A / I=H^{G_{m}}$, since a point of $H$ valued at $B, h: A \rightarrow B$ (morphism of $k$-algebras), is a fixed point if and only if $h$ is a morphism of $G_{m}$-algebras, where $B$ is endowed with the trivial structure of $G_{m}$-algebra; in other words, if and only if it is a morphism of graded $k$-algebras with the trivial graduation on $B\left(B=B_{0}\right)$. But this is equivalent to saying that $h(I)=0$, i.e., $h$ factors through $A / I$ or $h$ is a point of $\operatorname{Spec} A / I$ valued at $B$. This yields the existence of $\rho$ and proves that it is a morphism of schemes in groups.

The uniqueness is given from the fact that if a morphism $\rho: A / I \rightarrow A$ is a section of algebras and $G_{m}$-algebras of the projection $\pi: A \rightarrow A / I$, then it maps into $A_{0}$ (since it is a morphism of $G_{m}$-algebras) and, since it is a section of $\pi$, it must be the inverse of the isomorphism $\pi_{\left.\right|_{A_{0}}}: A_{0} \rightarrow A / I$.

(2) It is immediate that the action is conic. For the second part, in the above reduction, the retract is given by the inclusion $A_{0} \subset A$; if $A$ is the $\operatorname{ring}$ of $H$ and $\bar{A}$ the ring of $\bar{H}$, then the statement follows from the commutativity of the diagram

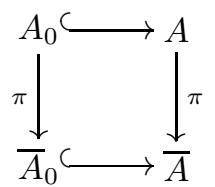

Corollary 2.12. With the notation of Corollary [2.10, one has that $U_{i}^{-}$is a subgroup of $U^{-}$, and it is the kernel of the unique retract of groups and $T$-schemes $\rho_{i-1}: U_{i-1}^{-} \rightarrow G_{a}^{\alpha_{i-1}}$.

Proof. (By recurrence on $i$ ). One has that $U_{i-1}^{-}$is a subgroup of $U^{-}$. Let us consider the subgroup $G_{m}^{(i-1)} \subset T$ of Lemma 2.9. From Corollary 2.10 one has that $\left(U_{i-1}^{-}\right)^{G_{m}^{(i-1)}}=G_{a}^{\alpha_{i-1}}$ and, by Proposition [2.11 there exists a unique retract $\rho_{i-1}: U_{i-1}^{-} \rightarrow G_{a}^{\alpha_{i-1}}$ of $G_{m}^{(i-1)}$-schemes which is, in addition, a morphism of groups. Therefore, it must coincide with the projection on $G_{a}^{\alpha_{i-1}}$, and hence $\operatorname{ker} \rho_{i-1}=U_{i}^{-}$; in particular, $U_{i}^{-}$is a subgroup of $U^{-}$and $\rho_{i-1}$ is a morphism of $T$-schemes.

Proposition 2.13. The subschemes in groups $H$ of $U_{S}^{-}$stable by $T$ are subschemes of the form

$$
H=H_{1} \underset{S}{\times} \cdots \underset{S}{\times} H_{s} \subset U_{S}^{-}
$$

with $H_{i} \subset\left(G_{a}^{\alpha_{i}}\right)_{S}$ a subscheme in groups stable by homotheties.

Proof. In order to simplify the notation, let us assume that $S=\operatorname{Spec} k$, i.e., $H \subset$ $U^{-}$, since the proof does not depend on the base $S$. Let us denote $T_{1}=G_{m}^{(1)} \subset T$. By Corollary 2.10, $U^{-}$and $H$ are cones with respect to $T_{1}$, and the fixed points are respectively $\left(U^{-}\right)^{T_{1}}=G_{a}^{\alpha_{1}}$ and $H^{T_{1}}=H \cap\left(U^{-}\right)^{T_{1}}=H \cap G_{a}^{\alpha_{1}}=H_{1}$. Moreover, the retract $\rho: U^{-} \rightarrow G_{a}^{\alpha_{1}}$ of $T_{1}$-schemes in groups restricts to the corresponding retract $\rho_{\left.\right|_{H}}: H \rightarrow H_{1}$ (Proposition [2.7). Thus, since ker $\rho=U_{2}^{-}$, one concludes that $H=H_{1} \times \bar{H}$ with $H_{1} \subset G_{a}^{\alpha_{1}}$ and $\bar{H} \subset U_{2}^{-}$. Recurrently, one concludes. 


\section{Proposition 2.14.}

(1) If $S$ is a connected scheme, then the subschemes in groups of $U_{S}^{-}$flat over $S$ and stable by $T$ are constant; that is, they are all obtained from the subgroups of $U^{-}$by the base change $S \rightarrow \operatorname{Spec} k$.

(2) If $S$ is integral, then the subschemes in groups of $U_{S}^{-}$stable by $T$ and flat over $S$ are precisely the ones with constant dimension and nilpotence degree along the fibres over $S$.

Proof. We may assume $S=\operatorname{Spec} A$. By the preceding proposition, any subgroup $H$ of $U_{S}^{-}$stable by $T$ (without flatness conditions over $S$ ) has the form $H_{1} \underset{S}{\times} \cdots \underset{S}{\times} H_{s}$, with $H_{i} \subset\left(G_{a}^{\alpha_{i}}\right)_{S}$ a stable subgroup by $T$. We may therefore assume $U^{-}=G_{a}=$ Spec $k[Y]$ and $H \subset\left(G_{a}\right)_{S}$ stable by homotheties. In this case, $H=\operatorname{Spec} \bigoplus_{i} A / I_{i} \cdot Y^{i}$ for certain ideals $I_{i} \subset A$ satisfying $I_{0} \subset I_{1} \subset \cdots \subset I_{n} \subset \cdots$.

(1) If $H$ is flat over $S$, then the $A$-modules $A / I_{i}$ are also flat; thus, they are locally free with rank less than or equal to 1 , i.e., either $I_{i}=0$ or $I_{i}=A$. It is then clear that $H$ descends to $U^{-}$.

(2) Assume $S$ is integral. If $H \subset\left(G_{a}\right)_{S}$ has constant dimension 1 along the fibres, then $H=\left(G_{a}\right)_{S}$, since the equality holds in the fibres over $S$. Therefore, $\left(I_{i}\right)_{0}=S$ and hence $I_{i}=0$ (since $S$ is integral). If $H$ has constant dimension 0 along the fibres, then for each point $x \in S$ the nilpotence degree is the maximum index $i$ such that $\mathfrak{p}_{x} \supset I_{i}\left(\mathfrak{p}_{x} \subset A\right.$ being the prime ideal of the functions that are zero at $x$ ). Since the nilpotence degree is constant along the fibres, one has that either $\left(I_{i}\right)_{0}=S$ or $\left(I_{i}\right)_{0}=\emptyset$; that is, either $I_{i}=0$ or $I_{i}=A$ for each $i$, and one concludes.

Proof of Theorem 2.2. This follows from Lemma 2.4 and Proposition 2.14.

\section{TRIViality of the ACtions With COMPlete orbits}

Definition 3.1. Let $X$ be a variety on which an algebraic group $G$ acts and let $x \in X$. We shall call the nilpotence degree of the orbit of $x$ the nilpotence degree of its isotropy group: $\left|P_{x} /\left(P_{x}\right)_{\text {red }}\right|$.

Remark 3.2. In characteristic zero this notion is superfluous, since any group is smooth and hence the nilpotence degree of any orbit is 1 .

Theorem 3.3. Let $G$ be an affine, smooth and connected algebraic group acting on a smooth variety $X$. Assume that the orbits of the action are complete and that they have constant dimension and nilpotence degree. Then all the orbits are isomorphic as $G$-varieties to a given one $Y$ and $X \simeq Y \times X / G$ as $G$-varieties, where the action of $G$ on $X / G$ is trivial. In particular, $X / G$ is a geometric quotient of $X$ by $G$.

Proof. Choose $B$, a Borel subgroup. Let $X^{B}$ be the reduced subscheme of the fixed points of $X$ under the action of $B$. Then $B$ fixes a unique point in any $G$-orbit in $X$. So the map $G \times X^{B} \rightarrow X$ is surjective and its fiber at $x \in X^{B}$ is $G_{x}$ (i.e., the isotropy group of $x$ ), which is irreducible and of constant dimension (since the dimension of $G / G_{x}$ is constant). Thus, $X^{B}$ is irreducible.

Let us define $\phi: G \times X^{B} \rightarrow X \times X^{B}$ by $\phi(g, x)=(g \cdot x, x)$ and let $\widetilde{P}=$ $\phi^{-1}\left(\Delta_{X^{B}}\right) \subset G \times X^{B}$, where $\Delta_{X^{B}}=X^{B} \subset X \times X^{B}$ is the diagonal. It is a subscheme in groups of $G \times X^{B}$ over $X^{B}$; it contains $B \times X^{B}$, and its fibre in each 
point $x \in X^{B}$ is the isotropy group of $x$ that, by hypothesis, has constant dimension and nilpotence degree. Since $X^{B}$ is connected and integral, one concludes, by Theorem 2.2, that it is constant: $\widetilde{P}=\bar{P} \times X^{B}$, with $\bar{P} \subset G$ a subgroup containing $B$. It thus follows that $\varphi$ factors through an isomorphism $G / \bar{P} \times X^{B} \rightarrow X$.

\section{INVARIANT INVERTIBLE SHEAVES}

Definition 4.1. Let $X$ be a complete and homogeneous variety. An invertible sheaf $\mathcal{L} \in \operatorname{Pic}(X)$ is said to be invariant if it is a fixed point of $\operatorname{Pic}(X)$ under the natural action of $\operatorname{Aut}^{0}(X)$ in $\operatorname{Pic}(X)$.

Remark 4.2. If $X$ is a parabolic variety, then any invertible sheaf is invariant, since $P i c_{r e d}(X)$ is a discrete $k$-scheme (because $\operatorname{Pic}^{0}(X)=0$ ) and $\operatorname{Aut}^{0}(X)$ is smooth and connected.

Proposition 4.3. Let $X$ be a complete and homogeneous variety. If $\mathcal{L} \in \operatorname{Pic}(X)$ is effective and invariant, then the complete linear system $\Gamma(X, \mathcal{L})$ has no base points, $\operatorname{Aut}^{0}(X)$ acts by automorphisms on $\mathbb{P}\left(H^{0}(X, \mathcal{L})^{*}\right)$, and the natural morphism $\phi_{\mathcal{L}}: X \rightarrow \mathbb{P}\left(H^{0}(X, \mathcal{L})^{*}\right)$ is an $\operatorname{Aut}^{0}(X)$-morphism.

Proof. Let $G=\operatorname{Aut}^{0}(X)$, and let $m: G \times X \rightarrow X$ be the action. Since $\mathcal{L}$ is invariant, one has $m^{*} \mathcal{L} \simeq \mathcal{L}_{G} \underset{k}{\otimes} \mathcal{L}$, for some invertible sheaf $\mathcal{L}_{G}$ on $G$. Taking direct images over $G$, one obtains $1 \otimes m^{*}: \mathcal{O}_{G} \underset{k}{\otimes} H^{0}(X, \mathcal{L}) \stackrel{\sim}{\rightarrow} \mathcal{L}_{G} \underset{k}{\otimes} H^{0}(X, \mathcal{L})$, and hence a morphism of $G$-schemes

$$
G \times \mathbb{P}\left(H^{0}(\mathcal{L})\right)=\mathbb{P}_{G}\left(\mathcal{O}_{G} \underset{k}{\otimes} H^{0}(\mathcal{L})\right) \stackrel{1 \otimes m^{*}}{\longrightarrow} \mathbb{P}_{G}\left(\mathcal{L}_{G} \underset{k}{\otimes} H^{0}(\mathcal{L})\right)=G \times \mathbb{P}\left(H^{0}(\mathcal{L})\right),
$$

where we have denoted $H^{0}(\mathcal{L})=H^{0}(X, \mathcal{L})$. Taking a fibre at each point of $G$, one obtains a morphism $\tau: G \rightarrow \operatorname{Aut}_{k}\left(\mathbb{P}\left(H^{0}(X, \mathcal{L})\right)\right)=\mathbf{P G l}_{k}\left(H^{0}(X, \mathcal{L})\right)$ such that for any rational point $g \in G$ and any section $s \in H^{0}(X, \mathcal{L})$ one has $\tau_{g}(\langle s\rangle)=\left\langle g^{*} s\right\rangle \subset$ $H^{0}\left(X, g^{*} \mathcal{L}\right)=H^{0}(X, \mathcal{L})$. Hence the transposed morphism $\bar{\tau}: G \rightarrow \mathbf{P G l}_{k}\left(H^{0}(X, \mathcal{L})^{*}\right)$ satisfies $\bar{\tau}_{g}(\langle\omega\rangle)=\langle g \cdot \omega\rangle$, for any $g \in G$ and $\omega \in H^{0}(X, \mathcal{L})^{*}$, where $(g \cdot \omega)(s)=$ $\omega\left(g^{*} s\right)$ for each $s \in H^{0}(X, \mathcal{L})$. It follows easily that $\bar{\tau}$ is a morphism of groups (notice that a morphism between two smooth groups is a morphism of groups if and only if it is so for the rational points). Moreover, if for each point $x \in X$ we denote by $\omega_{x}: H^{0}(X, \mathcal{L}) \rightarrow \mathcal{L}_{x} \simeq k$ the linear form that maps each section to its fibre at $x$, then $\left\langle g \cdot \omega_{x}\right\rangle=\left\langle\omega_{g \cdot x}\right\rangle$. Therefore, the closed subset of base points is empty, since it is $G$-invariant, $\mathcal{L}$ is effective, and $X$ is homogeneous, and the natural morphism $\phi_{\mathcal{L}}: X \rightarrow \mathbb{P}\left(H^{0}(X, \mathcal{L})^{*}\right)$, defined by $\phi_{\mathcal{L}}(x)=\left\langle\omega_{x}\right\rangle$, is a $G$-morphism.

Remark 4.4. This theorem implies the following well-known results:

(1) If $X$ is an abelian variety, $\mathcal{L} \in P i c^{0}(X)$ is nontrivial if and only if $H^{0}(X, \mathcal{L})=$ 0 .

(2) A complete and homogeneous variety $X$ is parabolic if and only if $\operatorname{Aut}^{0}(X)$ is an affine group (and, in that case, it is semisimple of adjoint type).

Definition 4.5. We shall say that a morphism $\pi: X \rightarrow Y$ of smooth varieties is a quotient of $X$ if

(1) $\pi$ is surjective (as a map),

(2) $\pi$ is submersive, i.e., a subset $U \subset Y$ is open if and only if $\pi^{-1}(U)$ is open in $X$, 
(3) $\mathcal{O}_{Y}=\operatorname{ker}(I d \otimes 1-1 \otimes I d)$, with $I d \otimes 1-1 \otimes I d: \pi_{*} \mathcal{O}_{X} \rightarrow \pi_{*} \mathcal{O}_{X} \underset{\mathcal{O}_{Y}}{\otimes} \pi_{*} \mathcal{O}_{X}$ the natural morphism.

We shall say that a quotient $\pi: X \rightarrow Y$ is an invariant quotient if (4) there exists an action of $\operatorname{Aut}^{0}(X)$ on $Y$ such that $\pi$ is a $\operatorname{Aut}^{0}(X)$-morphism.

This notion of quotient corresponds to the notion of quotient by an equivalence relation in the theory of schemes; that is, it is wider than Mumford's notion of geometric quotient under the action of a group.

Remark 4.6. If $X$ is a homogeneous variety, then one morphism $\pi: X \rightarrow Y$ (where $Y$ is a smooth variety) is an invariant quotient of $X$ if and only if it is surjective and invariant (i.e., it satisfies the conditions (1) and (4)). Indeed, if $\pi$ is invariant, then the fibers are equidimensional and so $\pi$ is flat ( $X, Y$ are smooth varieties). Moreover, if $\pi$ is surjective, then $\pi$ is faithful flat, and one concludes easily, by flat descent, that $\pi$ satisfies conditions (2) and (3).

Definition 4.7. We shall say that two quotients $\pi: X \rightarrow Y, \pi^{\prime}: X \rightarrow Y^{\prime}$ are equivalent if there exists an isomorphism $\phi: Y \rightarrow Y^{\prime}$ such that $\pi^{\prime}=\phi \circ \pi$.

Remark 4.8. Let $X$ be a homogeneous variety. An invariant quotient $\pi: X \rightarrow Y$ is determined, up to equivalence, by the fibre passing through a given point $x \in X$, that is, by the subscheme $\pi^{-1}(\pi(x)) \subset X$. Indeed, if $G=\operatorname{Aut}^{0}(X)$ and $H$ is the isotropy group of $x$, then $X=G / H, Y=G / H^{\prime}$ (with $H^{\prime}$ the isotropy group of $\pi(x))$ and $\pi^{-1}(\pi(x))=H^{\prime} / H \subset G / H$.

Remark 4.9. The invariant quotients of $X$, up to equivalence, are partially ordered in the following way: $(Y, \pi) \geq\left(Y^{\prime}, \pi^{\prime}\right)$ if there exists a morphism $h: Y \rightarrow Y^{\prime}$ of $\operatorname{Aut}^{0}(X)$-varieties such that $\pi^{\prime}=h \circ \pi$. Moreover, they form an inverse system: if $\left(Y_{1}, \pi_{1}\right),\left(Y_{2}, \pi_{2}\right)$ are two invariant quotients, there exists a third one, $(Y, \pi)$, such that $(Y, \pi) \geq\left(Y_{1}, \pi_{1}\right),\left(Y_{2}, \pi_{2}\right)$; it is enough to define $Y$ as the image of the $\operatorname{Aut}^{0}(X)$-morphism $\pi_{1} \times \pi_{2}: X \rightarrow Y_{1} \times Y_{2}$. Notice that if $Y_{1}$ and $Y_{2}$ are parabolic varieties (respectively, abelian varieties), then $Y$ is parabolic (respectively, abelian).

Lemma 4.10. Let $X$ be a complete variety, $\mathcal{L}$ an invertible sheaf on $X$ and $V_{2} \subset$ $V_{1} \subset H^{0}(X, \mathcal{L})$ two linear systems without base points. Let $\pi_{i}: X \rightarrow X_{i} \subset \mathbb{P}\left(V_{i}^{*}\right)$ be the morphism induced by $V_{i}$, with $X_{i}=\operatorname{Im} \pi_{i}$. There exists a morphism $h: X_{1} \rightarrow$ $X_{2}$ such that $\pi_{2}=h \circ \pi_{1}$. Moreover, $h$ is finite.

Proof. Let $\left(V_{2}\right)_{0} \subset V_{1}^{*}$ be the subspace incident with $V_{2}$. Let us consider the natural projection $\bar{h}: \mathbb{P}\left(V_{1}^{*}\right)-\mathbb{P}\left(\left(V_{2}\right)_{0}\right) \rightarrow \mathbb{P}\left(V_{2}^{*}\right)$. Then $\pi_{1}^{-1}\left(X_{1} \cap \mathbb{P}\left(\left(V_{2}\right)_{0}\right)\right)$ is the subscheme of base points of $V_{2}$, which is empty; hence $\bar{h}$ induces a morphism $h: X_{1} \rightarrow X_{2}$.

The finiteness of $h$ follows from the fact that it is an affine morphism, since $\bar{h}$ is affine.

Proposition 4.11. Let $X$ be a complete and homogeneous variety. There exists a parabolic (respectively, abelian) invariant quotient $\pi: X \rightarrow \mathcal{P}(X)$ satisfying the following universal property: for any parabolic (respectively, abelian) invariant quotient $\bar{\pi}: X \rightarrow \overline{\mathcal{P}}$, there exists a unique morphism $f: \mathcal{P}(X) \rightarrow \overline{\mathcal{P}}$ such that $\bar{\pi}=f \circ \pi$. Moreover, there exists an effective and invariant invertible sheaf $\mathcal{L}$ on $X$ such that $\mathcal{P}(X)=\operatorname{Proj} \bigoplus_{n \in \mathbb{N}} H^{0}\left(X, \mathcal{L}^{n}\right)$ and the natural morphism

$$
X \longrightarrow \operatorname{Proj} \bigoplus_{n \in \mathbb{N}} H^{0}\left(X, \mathcal{L}^{n}\right)=\mathcal{P}(X)
$$


coincides with $\pi$.

Proof. The invariant quotients are determined by the fibre through a given point $x$, in such a way that the order of the quotients corresponds with the inclusion order of the fibres; that is, $(Y, \pi) \geq(\bar{Y}, \bar{\pi})$ if and only if $\pi^{-1}(\pi(x)) \subset \bar{\pi}^{-1}(\bar{\pi}(x))$. Since $X$ is Noetherian, one concludes the existence of a maximal parabolic (respectively, abelian) quotient.

For the second part, let $\overline{\mathcal{L}}$ be an ample invertible sheaf on $\mathcal{P}(X)$. Since $\mathcal{P}(X)$ is parabolic, $\overline{\mathcal{L}}$ is invariant, and so $\mathcal{L}=\pi^{*} \overline{\mathcal{L}}$ is invariant too. Let us consider the parabolic quotient $\bar{\pi}: X \rightarrow \overline{\mathcal{P}}=\operatorname{Proj} \bigoplus_{n \in \mathbb{N}} H^{0}\left(X, \mathcal{L}^{n}\right)$. One has that $\mathcal{P}(X)=$ $\operatorname{Proj} \bigoplus_{n \in \mathbb{N}} H^{0}\left(\mathcal{P}(X), \overline{\mathcal{L}}^{n}\right)$ and $H^{0}\left(\mathcal{P}(X), \overline{\mathcal{L}}^{n}\right) \subset H^{0}\left(X, \mathcal{L}^{n}\right)$. This defines a morphism $h: \overline{\mathcal{P}} \rightarrow \mathcal{P}(X)$ such that $\pi=h \circ \bar{\pi}$ (Lemma 4.10). Hence $(\overline{\mathcal{P}}, \bar{\pi}) \geq(\mathcal{P}(X), \pi)$ and, by maximality, one concludes that $(\overline{\mathcal{P}}, \bar{\pi})=(\mathcal{P}(X), \pi)$.

Definition 4.12. The universal parabolic quotient $\pi: X \rightarrow \mathcal{P}(X)$ of the latter proposition is called the parabolic part of $X$. The universal abelian quotient $\pi_{a b}: X \rightarrow A b(X)$ is called the abelian part of $X$.

\section{Structure of COMPlete AND homogeneous VARIEties}

Lemma 5.1. Let $G$ be a smooth and connected group. If $G$ is a normal subgroup of a smooth and connected group $\bar{G}$ and $\bar{G}$ acts transitively on a variety $Y$, then the orbits of $Y$ under the action of $G$ are closed and conjugated by $\bar{G}$ (in particular, the orbits are isomorphic as $G$-schemes). In addition, there exists a fine quotient $Y \rightarrow Y / G$.

Proof. One has that $Y \simeq \bar{G} / H$, as $\bar{G}$-schemes. Since $G$ is normal in $\bar{G}$, it follows that $G \cdot H \subset \bar{G}$ is a subgroup and it is clear that $Y / G \simeq \bar{G} / G \cdot H$ as $\bar{G}$-schemes, in such a way that the morphism $\pi: Y \rightarrow Y / G$ corresponds to $\bar{G} / H \rightarrow \bar{G} / G \cdot H$. One concludes easily.

Theorem 5.2. If $X$ is a complete and homogeneous variety over $k$, then:

(1) $X$ splits, in a unique way, as the direct product of a parabolic variety and an abelian variety, $X=Y \times A$. These factors are canonically determined from $X$ in the following way: $A$ is the Albanese variety of $X$ (that is, $A=\operatorname{Pic}^{0}\left(\operatorname{Pic}^{0}(X)\right)$, where $P i c^{0}$ denotes the reduced connected component through the origin of the Picard scheme) and $\pi_{1}: X \rightarrow Y$ is the parabolic part of $X$.

(2) $X$ is projective, and (the connected and reduced component of) its automorphism scheme splits, in a unique way, as a direct product of an abelian variety and a semisimple group of adjoint type. More precisely: if $X=Y \times A$, with $Y$ the parabolic part and $A$ the abelian one, then $\operatorname{Aut}^{0}(X)=\operatorname{Aut}^{0}(Y) \times A$ and $\operatorname{Aut}^{0}(Y)$ is a semisimple group of adjoint type.

Proof. Let $\bar{G}=\operatorname{Aut}^{0}(X)$. Let $G \subset \bar{G}$ be the unique affine, smooth, connected and normal subgroup such that $A=\bar{G} / G$ is an abelian variety (Theorem 1.1). By Lemma $5.1 G$ acts on $X$ with closed and isomorphic (as $G$-schemes) orbits. Then, by Theorem [3.3, $X=G / P \times X / G$, and $A=X / G$ is a quotient of $\bar{G} / G=A$ and hence an abelian variety.

For the uniqueness of the decomposition $X=Y \times A$, it is enough to determine canonically the projections of $X$ onto the factors:

(1) Let $P i c^{0}$ be the reduced connected component through the origin of the Picard scheme of $X$, which is an abelian variety (Theorem 1.13(1)). It is known 
that $\operatorname{Pic}^{0}(Y)=0\left(\right.$ Theorem 1.13)(2)) and that $\operatorname{Pic}^{0}\left(\operatorname{Pic}^{0}(A)\right)=A$ (Theorem[1.14), and this equality is canonical up to translations of $A$ (that is, it is canonical once the origin is fixed). Moreover, $\operatorname{Pic}^{0}(Y \times A)=\operatorname{Pic}^{0}(Y) \oplus \operatorname{Pic}^{0}(A)$ (Theorem [1.15), and one thus has a natural morphism

$$
X \rightarrow \operatorname{Pic}^{0}\left(\operatorname{Pic}^{0}(X)\right)=\operatorname{Pic}^{0}\left(\operatorname{Pic}^{0}(Y) \times \operatorname{Pic}^{0}(A)\right)=\operatorname{Pic}^{0}\left(\operatorname{Pic}^{0}(A)\right)=A,
$$

which is precisely the projection. In other words, $A$ is the Albanese variety of $X$, and the projection $X \rightarrow A$ is the natural morphism $X \rightarrow A l b(X)$.

(2) In order to see that $\pi_{1}: X \rightarrow Y$ is the parabolic part of $X$, it is enough to see that $\left(Y, \pi_{1}\right) \geq(\mathcal{P}(X), \pi)$, i.e., that the fibre of a point $y_{0} \in Y$ by $\pi_{1}, y_{0} \times A \subset X$, is contained in some fibre of $\pi$ (Remark 4.8). By Proposition 4.11, $\pi: X \rightarrow \mathcal{P}(X)$ is the morphism induced by an effective invariant invertible sheaf $\mathcal{L}$ on $X$; hence it suffices to prove that $\mathcal{L}$ is trivial on $y_{0} \times A$. But, since $A=y_{0} \times A$ is an abelian variety, any effective invariant invertible sheaf is trivial (Remark 4.4 (1)). The restriction of $\mathcal{L}$ to $A$ is effective because $H^{0}(X, \mathcal{L}) \neq 0$ has no base points (Proposition 4.3).

For the second part of the statement, it is enough to observe that, since the projections onto the parabolic and abelian parts are invariant quotients, any automorphism induces an automorphism of each factor, and conversely. The rest is immediate.

Corollary 5.3. Any group extension of an abelian variety by a semisimple group of adjoint type is trivial.

Proof. Let $A$ be an abelian variety, $G$ a semisimple group of adjoint type, and let

$$
0 \rightarrow G \rightarrow \bar{G} \rightarrow A \rightarrow 0
$$

be a group extension. Let $B \subset G$ be a Borel subgroup of $G$ and $X=\bar{G} / B$. Since $G$ is a normal subgroup of $\bar{G}$, it follows easily that the orbits of $X$ under the action of $G$ are all isomorphic to $G / B$. Hence, by Theorem $3.3 \times=G / B \times A$ (since $\bar{G} / G=A$ ) and $\bar{G} \subset \operatorname{Aut}^{0}(X)=\operatorname{Aut}^{0}(G / B) \times \operatorname{Aut}^{0}(A)=\operatorname{Aut}^{0}(G / B) \times A$. Let $\pi_{1}: \operatorname{Aut}^{0}(G / B) \times A \rightarrow \operatorname{Aut}^{0}(G / B)$ be the natural projection. Then $\pi_{1}(G)=$ $\operatorname{Aut}^{0}(G / B)=G$ (Demazure [4] $)$. It follows that $\pi_{1}: \bar{G} \rightarrow G$ is a retract of groups, and hence $\bar{G}$ is the trivial extension.

Definition 5.4. The parabolic type of a homogeneous variety $X$ is the class, modulo automorphisms of $\operatorname{Aut}^{0}(X)$, of the isotropy group of any point of the variety under the action of its group of automorphisms (more properly, the reduced connected component of its group of automorphisms).

Remark 5.5. From the preceding theorem, the isotropy group of any point $x$ of a complete homogeneous variety $X$ is a parabolic subgroup of the affine part of its group of automorphisms. Therefore, the parabolic type of the variety coincides with the class of its parabolic part modulo isomorphisms.

Theorem 5.6. Two complete and homogeneous varieties are isomorphic if and only if their groups of automorphisms are isomorphic and they have the same parabolic type. In particular, the group of automorphisms classifies the variety, once its parabolic type has been given. In characteristic zero, there exist at most a finite number of complete homogeneous varieties with a given group of automorphisms. 
Given a complete and homogeneous variety $X=Y \times A$, let us denote by $\operatorname{Aut}^{l i n}(X)$ the maximum affine, normal, smooth and connected algebraic subgroup of $\operatorname{Aut}^{0}(X)$ (as usual, $\operatorname{Aut}^{0}(X)$ denotes the reduced connected component of the group of automorphisms of $X)$, and let $\operatorname{Aut}^{A b}(X)=\operatorname{Aut}^{0}(X) / \operatorname{Aut}^{l i n}(X)=$ $A l b(X)$.

Theorem 5.7 (Classification of complete homogeneous varieties). The classification of complete homogeneous varieties is equivalent to the classification of the triplets $(A, G,[P])$, where $A$ is an abelian variety, $G$ is a semisimple group of adjoint type, and $[P]$ is a parabolic type of $G$ such that $G=\operatorname{Aut}^{0}(G / P)$. The correspondence is

$$
\begin{aligned}
X & \mapsto\left(A l b(X), \operatorname{Aut}^{l i n}(X),\left[P_{x}\right]\right), \\
A \times G / P & \leftarrow(A, G,[P]),
\end{aligned}
$$

where $P_{x} \subset \operatorname{Aut}^{l i n}(X)$ is the isotropy group of any point $x \in X$ and $\left[P_{x}\right]$ denotes its class modulo automorphisms of $\operatorname{Aut}^{\text {lin }}(X)$.

\section{Classification of parabolic varieties}

To conclude, we are going to give the classification of the parabolic varieties from the following results:

- The classification of the parabolic subgroups given by Wenzel in [12] (in characteristic different from 2 and 3 ).

- The determination (given by Demazure in [4]) of the pairs $P \subset G$, where $G$ is a simple group of adjoint type and $P \subset G$ is a reduced parabolic subgroup such that $G=\operatorname{Aut}^{0}(G / P)$. The pairs satisfying this condition are called non-exceptional. Demazure proves that the exceptional pairs are the following ones:

(1) $G=\mathbf{S O}_{2 l+1}(k)$ and $G / P$ the variety that parameterizes the totally isotropic subspaces $V_{l} \subset k^{2 l+1}$ (with $2 l+1 \geq 5$ ). In this case $\operatorname{Aut}^{0}(G / P) \simeq \mathbf{P S O}_{2 l+2}$.

(2) $G=\mathbf{S p}_{2 l}(k)$ and $G / P=\mathbb{P}_{2 l-1}$ the variety that parameterizes the lines of $k^{2 l}$. In this case $\operatorname{Aut}^{0}\left(\mathbb{P}_{2 l-1}\right) \simeq \mathbf{P G l}_{2 l}(k)$.

(3) $G=$ the simple group of adjoint type with semisimple rank 2 and type $G_{2}$; that is, it is the group of automorphisms of an algebra of octonions $\Omega$. Let $\widetilde{\Omega} \subset \Omega$ be the hyperplane of the pure octonions and $G / P$ the variety of isotropic lines of $\widetilde{\Omega}$. Then $G / P$ is isomorphic to a projective quadric of dimension 5, and hence, $\operatorname{Aut}^{0}(G / P)=\mathbf{P S O}_{6}(k)$.

Theorem 6.1. A complete homogeneous variety $X$ splits as a direct product of two varieties $X=Y_{1} \times Y_{2}$ if and only if the group of automorphisms $\operatorname{Aut}^{0}(X)$ splits as a direct product of two groups $\operatorname{Aut}^{0}(X)=G_{1} \times G_{2}$. Moreover, one has:

(1) $Y_{1}, Y_{2}$ are complete and homogeneous varieties.

(2) $\mathcal{P}(X)=\mathcal{P}\left(Y_{1}\right) \times \mathcal{P}\left(Y_{2}\right)$.

(3) $A b(X)=A b\left(Y_{1}\right) \times A b\left(Y_{2}\right)$.

(4) $\operatorname{Aut}^{0}(X)=\operatorname{Aut}^{0}\left(Y_{1}\right) \times \operatorname{Aut}^{0}\left(Y_{2}\right)$.

Proof. Assume that $X=Y_{1} \times Y_{2}$. Since $Y_{1} \simeq Y_{1} \times y_{2} \hookrightarrow X$ for any closed point $y_{2} \in$ $Y_{2}, Y_{1}$ is projective. Analogously, $Y_{2}$ is projective. Moreover, they are both reduced and connected, since the product $Y_{1} \times Y_{2}=X$ is so. In particular, $\operatorname{Pic}^{0}(X)=$ $\operatorname{Pic}^{0}\left(Y_{1}\right) \times P i c^{0}\left(Y_{2}\right)$. It follows that $A b(X)=A b\left(Y_{1}\right) \times A b\left(Y_{2}\right)$ and the morphism $\mu: X \rightarrow A b(X)$ is precisely $\mu=\mu_{1} \times \mu_{2}: X=Y_{1} \times Y_{2} \rightarrow A b\left(Y_{1}\right) \times A b\left(Y_{2}\right)$. On 
the other hand, the fibre by $\mu$ of any point $x=\left(y_{1}, y_{2}\right)$ is $\mathcal{P}(X)=F_{1} \times F_{2}$, where $F_{1} \subset Y_{1}, F_{2} \subset Y_{2}$ are the fibres by $\mu_{1}$ and $\mu_{2}$ of the points $y_{1}$ and $y_{2}$, respectively.

Analogously, one proves that $F_{1}, F_{2}$ are projective and connected varieties. Let $\overline{\mathcal{L}}_{1}$ be an effective ample invertible sheaf on $F_{1}$. Then $\mathcal{L}_{1}=\overline{\mathcal{L}}_{1} \underset{k}{\otimes \mathcal{O}_{F_{2}}}$ is an invertible sheaf on $\mathcal{P}(X)$, and hence invariant (since $\mathcal{P}(X)$ is a parabolic variety). The natural morphism

$$
\mathcal{P}(X) \rightarrow \operatorname{Proj} \bigoplus_{n \in \mathbb{N}} H^{0}\left(\mathcal{P}(X), \mathcal{L}_{1}^{n}\right)=\operatorname{Proj} \bigoplus_{n \in \mathbb{N}} H^{0}\left(F_{1}, \overline{\mathcal{L}}_{1}^{n}\right)=F_{1}
$$

is an invariant quotient, and it coincides with the projection on the first factor. One concludes that $F_{1}, F_{2}$ are $\operatorname{Aut}^{0}(\mathcal{P}(X))$-varieties and $\operatorname{Aut}^{0}(\mathcal{P}(X))=\operatorname{Aut}^{0}\left(F_{1}\right) \times$ $\operatorname{Aut}^{0}\left(F_{2}\right)$. In particular, one has that

$$
\begin{aligned}
\operatorname{Aut}^{0}(X)=\operatorname{Aut}^{0}(\mathcal{P}(X)) \times A b(X) \\
=\left(\operatorname{Aut}^{0}\left(F_{1}\right) \times A b\left(Y_{1}\right)\right) \times\left(\operatorname{Aut}^{0}\left(F_{2}\right) \times A b\left(Y_{2}\right)\right)=G_{1} \times G_{2} ;
\end{aligned}
$$

that is, $\operatorname{Aut}^{0}(X)$ splits as a direct product of groups in such a way that $G_{1}$ acts trivially on $Y_{2}$ and $G_{2}$ acts trivially on $Y_{1}$. So $\operatorname{Aut}^{0}(X)=G_{1} \times G_{2} \subset \operatorname{Aut}^{0}\left(Y_{1}\right) \times$ $\operatorname{Aut}^{0}\left(Y_{2}\right) \subset \operatorname{Aut}^{0}(X)$, and then $G_{1}=\operatorname{Aut}^{0}\left(Y_{1}\right)$ and $G_{2}=\operatorname{Aut}^{0}\left(Y_{2}\right)$.

Conversely, if $\operatorname{Aut}^{0}(X)=G_{1} \times G_{2}$, then $\operatorname{Aut}^{\text {lin }}(X)=G_{1}^{l i n} \times G_{2}^{l i n}$ and $\operatorname{Aut}^{A b}(X)=$ $G_{1}^{A b} \times G_{2}^{A b}$. In particular, $G_{1}^{\text {lin }}$ is a normal subgroup of $\operatorname{Aut}^{0}(X)$, and this is an affine smooth and connected group. By Lemma 5.1 $X=F_{1} \times X / G_{1}^{\text {lin }}$, and the projections are $\operatorname{Aut}^{0}(X)$-morphisms. $F_{1}$ is an orbit under the action of $G_{1}^{l i n}$; hence $G_{1}^{\text {lin }}$ is semisimple of adjoint type, $F_{1}=G_{1}^{\text {lin }} / P_{1}$ and the projection $X \rightarrow F_{1}$ is an $\operatorname{Aut}^{0}(X)$-morphism. Therefore, $G_{1}^{A b} \times G_{2}$ induces a group of automorphisms of $F_{1}$ that commute with $G_{1}^{\text {lin }}$, i.e., a subgroup of $\operatorname{Aut}_{G_{1}^{\text {lin }}-\text { var }}\left(G_{1}^{\text {lin }} / P_{1}\right)_{\text {red }}=$ $\left(N_{G_{1}^{\text {lin }}}\left(P_{1}\right) / P_{1}\right)_{\text {red }}=0$, where $N_{G_{1}^{\text {lin }}}\left(P_{1}\right)$ is the normalizer of $P_{1}$ in $G_{1}^{\text {lin }}$, because if $g \in G_{1}^{\text {lin }}$ normalizes $P_{1}$, then it normalizes $\left(P_{1}\right)_{\text {red }}$, and hence $g \in\left(P_{1}\right)_{\text {red }} \subset P_{1}$, since $N_{G}(P)=P$ for any reduced parabolic subgroup $P$ of an affine smooth and connected group $G$. Consequently, $\operatorname{Aut}^{0}\left(F_{1}\right)=G_{1}^{\text {lin }}$ and $\operatorname{Aut}^{0}\left(X / G_{1}^{\text {lin }}\right)=$ $G_{1}^{A b} \times G_{2}$. Repeating the same argument with $G_{2}^{\text {lin }}$ and $X / G_{1}^{\text {lin }}$, one obtains that $X / G_{1}^{l i n}=F_{2} \times X /\left(G_{1}^{\text {lin }} \times G_{2}^{\text {lin }}\right)=F_{2} \times A b(X)$, where the projections are invariant quotients, $\operatorname{Aut}^{0}\left(X / G_{1}^{\text {lin }}\right)=\operatorname{Aut}^{0}\left(F_{2}\right) \times \operatorname{Aut}^{0}(A b(X)), \operatorname{Aut}^{0}\left(F_{2}\right)=G_{2}^{\text {lin }}$ and $\operatorname{Aut}^{0}(A b(X))=\operatorname{Aut}^{A b}(X)=G_{1}^{A b} \times G_{2}^{A b}$. Regrouping the factors, one has $X=\left(F_{1} \times G_{1}^{A b}\right) \times\left(F_{2} \times G_{2}^{A b}\right)=Y_{1} \times Y_{2}$ and $\operatorname{Aut}^{0}\left(Y_{1}\right)=G_{1}, \operatorname{Aut}^{0}\left(Y_{2}\right)=G_{2}$.

Definition 6.2. We say that a homogeneous variety $X$ is indecomposable if it is not the product of two varieties of dimension greater than zero.

Corollary 6.3. A parabolic variety $X$ is indecomposable if and only if its automorphism group is a simple group of adjoint type.

Corollary 6.4. Any parabolic variety $\mathcal{P}$ splits uniquely (up to permutation of the factors) as a direct product of indecomposable parabolic varieties, $\mathcal{P}=\mathcal{P}_{1} \times \cdots \times \mathcal{P}_{r}$.

Proof. This follows from Theorem 6.1 and from the existence and uniqueness of the decomposition of an affine semisimple group of adjoint type as a product of simple groups. 
Remark 6.5. From Theorem 5.7 and Corollary 6.4, the classification of homogeneous varieties is reduced to the classification of abelian varieties and the classification of parabolic varieties with simple (of adjoint type) group of automorphisms.

Let $G$ be an affine, simple, algebraic group of adjoint type. Let $\mathcal{B}=G / B$. Then $G=\operatorname{Aut}^{0}(\mathcal{B})$ (see [4]), and any parabolic $G$-variety $\mathcal{P}$ is an invariant quotient $\mathcal{B} \rightarrow \mathcal{P}$ (in a unique way up to translations by elements of $G$ ). The problem is to determine the parabolic $G$-varieties $\mathcal{P}$ satisfying $\operatorname{Aut}^{0}(\mathcal{P})=G$.

First of all, let us specify what are the parabolic $G$-varieties, up to isomorphisms of varieties. Let us consider the associated parabolic varieties $\mathcal{P}_{1}=G / P_{1}, \ldots, \mathcal{P}_{s}=$ $G / P_{s}$, where $P_{1}, \ldots, P_{s}$ are the maximal reduced parabolic subgroups containing $B$.

Given two parabolic $G$-varieties $\pi: \mathcal{B} \rightarrow \mathcal{P}$ and $\pi^{\prime}: \mathcal{B} \rightarrow \mathcal{P}^{\prime}$, we shall denote by $\mathcal{P} * \mathcal{P}^{\prime}$ the parabolic $G$-variety image of the $G$-morphism $\pi \times \pi^{\prime}: \mathcal{B} \rightarrow \mathcal{P} \times \mathcal{P}^{\prime}$.

Remark 6.6. If $\mathcal{P}=G / P$ and $\mathcal{P}^{\prime}=G / P^{\prime}$ with $P, P^{\prime} \subset G$ parabolic subgroups containing $B$, then $\mathcal{P} * \mathcal{P}^{\prime}=G / P \cap P^{\prime}$. Moreover, $\mathcal{P} * \mathcal{P}^{\prime}=\sup \left(\mathcal{P}, \mathcal{P}^{\prime}\right)$, with respect to the order of the parabolic $G$-varieties.

Assume $\operatorname{char}(k)=p>0$. Given a variety $X$ and a natural number $n \in \mathbb{N}$, we shall denote by $X^{[n]}$ the scheme whose underlying topological space is $X$ and whose structural sheaf is the subsheaf $\mathcal{O}_{X}^{p^{n}} \subset \mathcal{O}_{X}$. One has a natural morphism of schemes $F^{n}: X \rightarrow X^{[n]}$ ( $F$ being the Frobenius morphism).

If $G$ is a scheme in groups, then $G^{[n]}$ is a scheme in groups and $F^{n}: G \rightarrow G^{[n]}$ is a morphism of groups. We shall denote $G_{n}=\operatorname{ker} F^{n}$, which is a subscheme in groups, finite and local.

Remark 6.7. It is easy to see that if $\mathcal{P}$ is a parabolic $G$-variety, then $\mathcal{P}^{[n]}$ is a parabolic $G$-variety too, and $\operatorname{Aut}^{0}(\mathcal{P}[n])=\operatorname{Aut}^{0}(\mathcal{P})^{[n]}$.

Theorem 6.8. Assume that $0<\operatorname{char}(k) \neq 2,3$. For each parabolic $G$-variety $\mathcal{P}$, there exist unique indices $1 \leq i_{1}<\cdots<i_{r} \leq s$ and exponents $n_{1}, \ldots, n_{r} \in \mathbb{N}$ such that

$$
\mathcal{P}=\mathcal{P}_{i_{1}}^{\left[n_{1}\right]} * \cdots * \mathcal{P}_{i_{r}}^{\left[n_{r}\right]} .
$$

Moreover, $\operatorname{Aut}^{0}(\mathcal{P})=G$ if and only if $n_{h}=0$ for some $1 \leq h \leq r$ and $\mathcal{P}_{i_{j}}$ is non-exceptional for some $1 \leq j \leq r$. That is, $G \rightarrow \operatorname{Aut}^{0}(\mathcal{P})$ is not an isomorphism if and only if either $n_{1}, \ldots, n_{r}>0$ or $P$ is maximal and $P \subset G$ is an exceptional pair.

Proof. With the notation of [12], one has that each parabolic subgroup $P$ of $G$ containing $B$ can be expressed in a unique way as $P=\bigcap_{\beta_{i} \in \mathcal{S}} P_{n_{i}, \beta_{i}}$, where $\mathcal{S}$ is the basis of the root system of $G$ corresponding to $T \subset B, P_{\beta_{i}}$ is the maximal parabolic subgroup such that the root system of $\left(P_{\beta_{i}}, T\right)$ does not contain $-\beta_{i}$, and $P_{n_{i}, \beta_{i}}=G_{n_{i}} \cdot P_{\beta_{i}}$ if $n_{i} \neq \infty$, and $P_{\infty, \beta_{i}}=G$. With our notation, $G / P_{\beta_{i}}=\mathcal{P}_{i}$, $G / P_{n_{i}, \beta_{i}}=\mathcal{P}_{i}^{\left[n_{i}\right]}$ if $n_{i} \neq \infty$, and $G / P_{\infty, \beta_{i}}=\operatorname{Spec} k$; then $\mathcal{P}=G / P=\mathcal{P}_{1}^{\left[n_{1}\right]} * \cdots *$ $\mathcal{P}_{s}^{\left[n_{s}\right]}$, where $\mathcal{P}_{i}^{[\infty]}=$ Spec $k$, i.e., it is a factor that can be suppressed.

For the second part, one observes that $G \rightarrow \operatorname{Aut}^{0}(\mathcal{P})$ is injective if and only if $\inf \left(n_{1}, \ldots, n_{r}\right)=0$, since, on the contrary, it factors through $G^{[1]}$. Moreover, it is surjective if and only if there exists a morphism $\pi$ : $\operatorname{Aut}^{0}(\mathcal{P}) \rightarrow G^{[n]}$ that coincides with $F^{n}$ over $G$. Indeed, if $\pi$ exists, then $(\operatorname{ker} \pi)^{0}$ is a normal subgroup 
of $\operatorname{Aut}^{0}(\mathcal{P})$, and $(\operatorname{ker} \pi)^{0} \cap G=\{e\}$. Hence, since $\operatorname{Aut}^{0}(\mathcal{P})$ is simple, one concludes that $(\operatorname{ker} \pi)^{0}=\{e\}$, and then $\operatorname{dim} \operatorname{Aut}^{0}(\mathcal{P})=\operatorname{dim} G^{[n]}=\operatorname{dim} G$; hence $G=\operatorname{Aut}^{0}(\mathcal{P})$. Now, if $\left(G, P_{i}\right)$ is non-exceptional, then $\operatorname{Aut}^{0}\left(\mathcal{P}_{i}\right)=G$. So (by the following lemma) one has $\operatorname{Aut}^{0}(\mathcal{P}) \rightarrow \operatorname{Aut}^{0}\left(\mathcal{P}_{i}^{\left[n_{i}\right]}\right)=\operatorname{Aut}^{0}\left(\mathcal{P}_{i}\right)^{\left[n_{i}\right]}=G^{\left[n_{i}\right]}$ and one concludes. Conversely, if $P$ is maximal and the pair $P \subset G$ is exceptional, then $G \varsubsetneqq \operatorname{Aut}^{0}(\mathcal{P})$.

Lemma 6.9. With the notation of the preceding theorem, the projections $\mathcal{P} \rightarrow \mathcal{P}_{i}^{\left[n_{i}\right]}$ are invariant quotients.

Proof. Let us consider the $G$-morphism $\pi_{i}: \mathcal{P} \rightarrow \mathcal{P}_{i}^{\left[n_{i}\right]}$, let $\overline{\mathcal{L}}$ be a very ample invertible sheaf on $\mathcal{P}_{i}^{\left[n_{i}\right]}$ and $\mathcal{L}=\pi_{i}^{*} \overline{\mathcal{L}}$. Let $\bar{\pi}: \mathcal{P} \rightarrow \overline{\mathcal{P}}$ be the invariant quotient defined by the complete linear system $H^{0}(\mathcal{P}, \mathcal{L})$ (Proposition 4.3). It is clear that $\bar{\pi}$ factors through a morphism $h: \overline{\mathcal{P}} \rightarrow \mathcal{P}_{i}^{\left[n_{i}\right]}$ and $h^{*} \overline{\mathcal{L}}$ is a very ample invertible sheaf on $\overline{\mathcal{P}}$; hence $h: \overline{\mathcal{P}} \rightarrow \mathcal{P}_{i}^{\left[n_{i}\right]}$ is a finite morphism with connected fibers (since these fibers are quotients of parabolic subgroups of $G$ ). Then $\overline{\mathcal{P}}=\mathcal{P}_{i}^{[n]}$ and, by the preceding theorem (first part), $n \geq n_{i}$. It is clear that $\mathcal{P}=\mathcal{P}_{1}^{\left[n_{1}\right]} * \cdots * \mathcal{P}_{i}^{[n]} * \cdots * \mathcal{P}_{s}^{\left[n_{s}\right]}$; hence, again by the first part of the preceding theorem, $n=n_{i}$; that is, $\overline{\mathcal{P}}=\mathcal{P}_{i}^{[n]}$ and $\pi_{i}=h$ is an invariant quotient.

Let $G$ be a simple algebraic group (of adjoint type) and $\mathcal{D}_{G}$ its Dynkin diagram. It is a connected graph (the Coxeter graph, whose vertices are the elements of a basis of roots of $G$ ), where each edge has a weight $m_{i}=1,2,3$. One has that $\operatorname{Aut}(G) / \operatorname{Inn}(G)=\operatorname{Aut}\left(\mathcal{D}_{G}\right)$, i.e., the automorphisms of $G$, modulo inner automorphisms, are the automorphisms of the graph $\mathcal{D}_{G}$ leaving the weights invariant. Moreover, one has that $\operatorname{Aut} G=\operatorname{Inn}(G) \rtimes \operatorname{Aut}\left(\mathcal{D}_{G}\right)$, where the identification $\operatorname{Aut}\left(\mathcal{D}_{G}\right) \subset$ Aut $G$ is given by the automorphisms of $G$ that leave invariant a given Borel subgroup $B$ and a given maximal torus $T \subset B$. Therefore, $\operatorname{Aut}\left(\mathcal{D}_{G}\right)$ acts on the set of parabolic subgroups containing $B$. The action is as follows: the vertices of $\mathcal{D}_{G}$ correspond with the elements of a basis $\mathcal{S}$ of roots of $G$, and these correspond with the reduced and maximal parabolic subgroups of $G$ containing $B, P_{1}, \ldots, P_{s}$. On the other hand, the parabolic subgroups of $G$ containing $B$ correspond biunivocally with the functions $\phi: \mathcal{S} \rightarrow \mathbb{N} \cup \infty$, i.e., with $\mathbb{N}^{*} \times \cdots \times \mathbb{N}^{*}=\mathbb{N}^{* \mathcal{S}}$, where $\mathbb{N}^{*}=\mathbb{N} \cup \infty$ and $\operatorname{Aut}\left(\mathcal{D}_{G}\right)$ acts on $\mathcal{S}$. Finally, for $G$ fixed, the parabolic $G$-varieties, modulo isomorphisms of varieties, correspond with the parabolic subgroups of $G$, modulo automorphisms of $G$, i.e., with the parabolic subgroups of $G$ containing $B$, modulo $\operatorname{Aut}\left(\mathcal{D}_{G}\right)$.

In conclusion, in characteristic different from 2 and 3, one has:

Theorem 6.10 (Classification of indecomposable parabolic varieties). Let $G$ be a simple algebraic group (of adjoint type), $\mathcal{S}$ the basis of roots of $G$ corresponding to $T \subset B \subset G$, and $\mathcal{D}_{G}$ its Dynkin diagram. The set of parabolic varieties whose automorphism group is isomorphic to $G$, modulo isomorphisms of varieties, is identified with the subset of the set

$$
\{\text { Parabolic } G \text {-Varieties }\} / \sim \stackrel{\sim}{\longrightarrow} \mathbb{N}^{* \mathcal{S}} / \operatorname{Aut}\left(\mathcal{D}_{G}\right)
$$

formed by the classes of elements $\left(n_{1}, \ldots, n_{s}\right) \in \mathbb{N}^{* \mathcal{S}}$ such that $n_{i}=0$ for some $i$ and $n_{j} \neq \infty$ for some $j$ such that $P_{j} \subset G$ is non-exceptional. 


\section{ACKNOWLEDGMENTS}

The author thanks the referee for valuable comments and suggestions.

\section{REFERENCES}

1. Borel, A. Symmetric Compact Complex Spaces. Arch. Math. (Basel) 33 (1979/80), no. 1, 49-56. MR 80k:32033

2. Borel, A. and Remmert, R. Über kompakte homogene Kählersche Mannigfaltigkeiten. Math. Ann. 145 (1961/1962), no. 1, 429-439. MR 26:3088

3. Chevalley, C. Séminaire sur la Classification des Groupes de Lie Algébriques. Paris: Ecole Norm. Sup. 1956-1958. MR 21:5696

4. Demazure, M. Automorphismes et Déformations des Variétés de Borel. Invent. Math. 39, 179-186 (1977). MR 55:8054

5. Grothendieck, A. Technique de descente et théorèmes d'existence en géométrie algébrique. V: Les schémas de Picard: Théorèmes d'existence, Séminaire Bourbaki, 14ième année, 1961/62, fasc. 3, Exposé 232, Secrétariat Math., Paris, 1962, and reprints. MR 26:3561 MR 33:5420i] MR 99f:00039

6. Humphreys, J. E. Linear Algebraic Groups. Graduate Texts in Mathematics 21, SpringerVerlag, New York (1975). MR 53:633

7. Matsumura, H. and Oort, F. Representability of Group Functors, and Automorphisms of Algebraic Schemes. Invent. Math. 4, 1-25 (1967). MR 36:181

8. Mumford, D. Abelian Varieties. Tata Studies in Math., Oxford Univ. Press (1970). MR 44:219

9. Mumford, D. On the Equations Defining Abelian Varieties, I, II, III. Invent. Math. 1 (1966), 287-354; 3 (1967), 76-135, 215-244. MR 34:4269; MR 36:2621 MR 36:2622

10. Mumford, D. and Fogarty, J. Geometric Invariant Theory. Springer-Verlag (1982). MR 86a:14006

11. Rosenlicht, M. Some Basic Theorems on Algebraic Groups. Amer. J. of Math. 78, 427-443, (1956). MR 18:514a

12. Wenzel, Ch. Classification of all Parabolic Subgroup-Schemes of a Reductive Linear Algebraic Group over an Algebraically Closed Field. Trans. Amer. Math. Soc. 337, 211-218 (1993). MR 93g:20090

Departamento de Matemáticas, Universidad de Salamanca, Plaza de la Merced 3-4, C.P. 37008 , EspañA

E-mail address: sancho@gugu.usal.es 